\title{
Für eine verantwortungsvolle Nationale Gesundheitspolitik!
}

\author{
Anmerkungen und Überlegungen der Ständigen Kommission für Strategie und Planung Psychiatrie und Psychotherapie \\ (SKSP) der Foederatio Medicorum Psychiatricorum et Psychotherapeuticorum (FMPP) ${ }^{1}$ zur Vernehmlassung des Projekts \\ Nationale Gesundheitspolitik Schweiz - Psychische Gesundheit
}

R. Raggenbass ${ }^{a}$

Das vorliegende Projekt Nationale Gesundheitspolitik - Psychische Gesundheit verdient eine kritische Würdigung. Einige Determinanten, die in den üblichen Diskussionen um die Fragen der psychischen Gesundheit selten bis gar nicht auftauchen, sollten hervorgehoben werden. Es gilt aufmerksam zu machen auf die Gefahren einer Politik, deren Blick sich zunehmend nach gewissen Prinzipien der Ökonomie ausrichtet, sowie einer psychiatrisch-psychotherapeutischen Versorgung, die sich - auf Kosten der klinischen Praxis - zunehmend am wissenschaftlichen Ideal orientiert. Wir halten Vielfalt in den Ansätzen zur Förderung der psychischen Gesundheit und Versorgung für richtig und erachten eine bessere Vernetzung der vorhandenen Strukturen als notwendig; wir befürworten im Bereich der psychischen Gesundheit die Schaffung eines ständigen multidisziplinären Koordinationsorgans auf nationaler Ebene. Eine verantwortungsvolle Politik sollte jedem ermöglichen, das Seine zu tun, um ein angemessenes Verhältnis zwischen den Realitäten des einzelnen und den Bedürfnissen der Gemeinschaft zu erreichen.

La version française

a paru dans le $n^{\circ} 35 / 2004$

a Präsident $\mathrm{SKSP}^{2}$,

Vorstandsmitglied FMPP ${ }^{3}$

Vorstandsmitglied SGPP ${ }^{4}$.

Korrespondenz:

Dr. med. René Raggenbass

Rue du Nord 2

CH-1920 Martigny

\section{Vorbemerkung}

Seit dem Jahr 2000 möchte die öffentliche Hand angesichts der epidemiologischen Erhebungen dem Bedarf im Bereich der Psychiatrie und der psychischen Gesundheit der Bevölkerung einen grösseren Platz einräumen. ${ }^{5}$ Sie hat dabei nicht nur einen diesbezüglichen Bedarf festgestellt, sondern unterstützt, wie es der von uns untersuchte Bericht zeigt, das Bemühen um neue gesetzliche, wirtschaftliche und strukturelle Vorgaben. Das Dokument ist das Ergebnis multidisziplinärer, breit angelegter und sehr konzentrierter Arbeit zu bereits Vorhandenem, benennt aber auch die Lücken, die im Bereich der Public Mental Health in der Schweiz noch auszumachen sind. Das Dokument ist derart dicht in seinen Analysen und anspruchsvoll in seinen Vorschlägen, dass es problematisch wird, sich kritisch dazu zu äussern und einzelne Prioritäten herauszuschälen. Diese Dichte ist Qualitätsmerkmal und Hauptmangel dieses Berichts zugleich. Er zielt auf das Ideale, doch weiss jeder in der psychiatrisch-psychotherapeutischen Versorgung Tätige, dass das Ideal gleichbedeutend mit dem Unmöglichen ist. Es geht somit darum - in Absprache mit den Akteuren vor Ort - realistische Mittel und Voraussetzungen zur Veränderung in diesem idealen Ganzen zu schaffen.

\section{Homo Faber - Träger und Schöpfer einer Ökonomie}

Es muss im Auge behalten werden, dass der Faktor «Ökonomie» das Schicksal sämtlicher Vorschläge bestimmen wird. Dies ist für uns Psychiater/-innen und Psychotherapeuten/-innen legitim, ja unvermeidbar. ${ }^{6}$ Eine zu politisch und wirtschaftlich ausgerichtete Lesart des Berichts birgt jedoch die Gefahr in sich, zu denken, ein Subjekt, das arbeitsfähig ist, sei gesund an Körper und Geist. Dies entspricht sicherlich nicht der Realität. Im Bericht heisst es denn auch: «Der Schutz, die Förderung, Erhaltung und Wiederherstellung der psychischen Gesundheit für die Bevölkerung muss weit über die Reduktion der Prävalenz und Inzidenz von psychischen Erkrankungen hinausgehen» (S. 62).

Wenn die Ökonomie - im weitesten Sinne, nicht im einseitig monetären Sinne klingender Münzen - eine für das Funktionieren jeglicher Gesellschaft unverzichtbare Hauptdeterminante ist, kann ihre finanzielle Bedeutung doch nicht Leitmotiv sein. Aus diesem Blickwinkel bedeutet Wirtschaft dann möglicherweise weniger das Schaffen und Verwalten von Gütern und Mehrwert als vielmehr eine Folge mehr oder weniger ausgeklügelter Strategien im Bemühen, die verschiedenen Formen des Mangels in Griff zu bekommen.

Es scheint uns, dass eine $\mathrm{zu}$ sehr auf das Monetäre ausgerichtete Politik im Bereich der psychischen Gesundheit und der psychiatrischpsychotherapeutischen Versorgung Politiker und Versicherer dazu drängt, ausschliesslich die Faktoren «Defizit» und «Rentabilität» zu berücksichtigen. Eine der spezifischen Eigenarten der psychischen Funktionsweise (der 
psychischen Ökonomie) und der psychischen Krankheit liegt aber genau darin, dass diese sich nicht auf eine Epidemiologie, die ausschliesslich auf einer Logik des Defizits beruht, reduzieren oder sich durch selbige erklären lassen. Aufgabe unserer nationalen Politik für Gesundheit und psychiatrisch-psychotherapeutische Versorgung ist es folglich, das Spezifische des Individuellen des Leidens (jeden Einzelfall) mit der Vielfalt der Interessen des Lebens in Gemeinschaft (soziale Einbettung) miteinander zu verbinden.

Die Anforderung, die die Politik an die Psychiatrie und Psychotherapie, aber auch an die anderen Akteure in der psychiatrisch-psychotherapeutischen Versorgung stellt, beschränkt sich bedauerlicherweise allzuoft darauf, die Defizite bzw. den Mangel (Mangel mit Blick auf das Dasein, die Arbeit, den Konsum) zu identifizieren, einzuordnen, zu berechnen und dann, so gut es eben geht, zu behandeln. Bei diesen wirtschaftlichen Vorgaben, denen die Akteure in der psychischen Behandlung zuweilen ohne es zu wollen folgen, läuft man in der Psychiatrie/Psychotherapie Gefahr, zu vergessen, dass die Aufgabe auch darin besteht, die allgemeine Ökonomie dieses Leidens für das Subjekt zu untersuchen. Eine solche Untersuchung vermittelt dem Subjekt in vielen Fällen, dass das scheinbare Defizit bzw. der Mangel ein positives Mittel darstellt, welches ihm ermöglicht, aktiv soziale Bindungen zu bewahren bzw. zu schaffen.

\section{Die Logik des «Defizits» ist kein Begriff aus der Psychiatrie / Psychotherapie, sondern ein Konzept aus der Ökonomie; wie kann das mit Versorgung der Bevölkerung und Public Health in Ein- klang gebracht werden?}

Der Begriff Defizit ist die Auslegung für einen «Mangel an ...» und hat den Doppelstatus eines (real) festgestellten Zustandes und den einer (imaginären oder symbolischen) Interpretation. Wenn er derart zum Handeln veranlasst, so deshalb, weil er sich auf ein Ideal bezieht; das Ideal von Einheit, eines permanenten Zugewinns, eines Zustands der Perfektion, von Nichtleiden, von Ungeteiltsein usw. Explizit - meist jedoch implizit - findet sich der Gedanke des Defizitären auch im vorliegenden Expertenbericht, insbesondere, wenn von fehlenden Infrastrukturen und Mitteln die Rede ist, vom Bemühen um Rehabilitation oder von Problemen rund um die Invalidenversicherung.
Die Antwort der Ökonomie auf den wahrnehmbaren Mangel ist eine ganz andere als die des Behandelnden. Die ökonomische Reaktion erfolgt unmittelbar und ist logischerweise an der Notwendigkeit ausgerichtet, rasch eine Lösung zu finden für etwas, was real wie auch scheinbar als Verlust oder Mangel interpretiert wird.

Der im Bereich der Versorgung tätige Psychiater/Psychotherapeut reagiert klinisch auf diese Beobachtung. Seine klinische Vorgehensweise besteht zuerst darin, im Einzelfall die dem Symptom des Patienten eigene Ökonomie zu untersuchen, zu identifizieren und dann das leidende Subjekt im Verständnis dafür, wie dieses Symptom/«Defizit» auch ein «Mehrwert» für es sein kann, zu unterstützen und zu begleiten. Bei einer bestimmten Anzahl von Personen, einer Minderheit, wird dies möglicherweise nicht gelingen. Bei diesem Personenkreis muss die Gemeinschaft auf die eine oder andere Weise intervenieren.

Für die Psychiatrie/Psychotherapie besteht die Gefahr, sich zu sehr vom wirtschaftlichen Kampf gegen das scheinbare Defizit bzw. den Mangel leiten zu lassen. Die Probleme stellen sich auf epistemologischer, ethischer und politischer Ebene. Auf epistemologischer Ebene besteht die Gefahr darin, sich nicht mehr mit den klinischen Mitteln zu versehen, mittels derer die ökonomische Rolle des Symptoms für das Subjekt und folglich auch für die Bevölkerung berücksichtigt werden kann. Eine solche Ausrichtung kann dazu führen, dass das Subjekt dazu gedrängt wird, sich zu ändern, ein anderes Symptom zu erfinden (hier wird das Subjekt immer eine Schrittlänge voraus sein), um in der gleichen ökonomischen Dynamik verbleiben zu können. So etwa geben uns die somatoformen Schmerzsyndrome unmittelbar zu denken. Je nach diesbezüglicher Antwort besteht paradoxerweise die Gefahr, diese Logik des Defizits weiter aufrechtzuerhalten, indem diagnostische Systeme, Behandlungen und Versorgungs- und Rehabilitationseinrichtungen dazu genutzt werden, dem Subjekt diesen Mehrwert, der beispielsweise in seiner Abhängigkeit vom System liegen kann, zu belassen. Aus ethischer Sicht hat diese Logik des Defizits zur Folge, dass tendenziell Patientengruppen gebildet werden, die vorschnell «gettoisiert» und stigmatisiert werden (z.B. Bezüger/innen einer Invalidenrente, Behinderte, Zwangsneurotiker, Personen mit Angstneurosen usw.) Durch ihre - ärztlich bescheinigte neue - Identität legitimiert, äussern oder formulieren solche Menschen nicht mehr einen 
Bedarf nach individueller Behandlung, um zu genesen oder einen angemessenen Umgang mit dem eigenen Symptom zu suchen, sondern fordern eine Entschädigung bzw. Kompensation für etwas, was wir vielleicht medizinisch fälschlicherweise als Defizit deklariert haben. Auf politischer Ebene schliesslich besteht die Gefahr, in dieser Logik eine Lösung zu sehen, die einem erspart, über die schwierige Komplexität der psychischen Ökonomie jeder Einzelsituation nachdenken zu müssen (kollektives Verneinen von Eigenverantwortung).

\section{Handeln im Sinne von Verbindung zwischen verantwortungsvollem Subjekt (Individuum) und Kollektiv (soziale Einbettung)}

Die Vorgehensweise in der Psychiatrie/Psychotherapie zielt sowohl darauf ab, das Subjekt nicht aus der Verantwortung zu entlassen ${ }^{7}$, als auch darauf, die Voraussetzungen, die das Subjekt im sozialen Gefüge halten, zu bewahren. Um dies $\mathrm{zu}$ erreichen, müssen Behandelnde, Politiker und Versicherer alles daran setzen, den Bestand, den Zugang und insbesondere die Vielfalt der Versorgungsstrukturen und -modalitäten zu sichern. Der Erhalt und die Förderung einer solchen Vielfalt sind der Preis für die soziale Einbettung. Gerade auf dieser Ebene zeichnen sich die meisten Spannungen zwischen der symbolischen und imaginären Logik des globalen Ansatzes (Epidemiologie, Makroökonomie usw.) und der realen Logik jedes Einzelfalles ab.

Um sich ein Bild von den gesundheitlichen, für unsere Gesellschaft relevanten Anforderungen machen zu können, ist es notwendig, statistische und epidemiologische Zahlen zu den psychischen Krankheiten heranzuziehen. Der selbe Zugang ist jedoch problematischer, wenn es um die psychiatrisch-psychotherapeutische Behandlung geht. Bei dieser sollte - wenn man verstehen will, worum es geht - nicht der Auftrag, sondern der Wunsch, der dahintersteht, wortwörtlich genommen werden. Psychiatrischpsychotherapeutische Behandlung kann nicht stattfinden oder durchgeführt werden, ohne den Wunschcharakter der Beziehung zum anderen zu berücksichtigen, wie er jedem einzelnen eigen ist. In der Behandlung haben wir mit Einzelfällen zu tun. Zwar ist es wichtig, dass die Leistungserbringer epidemiologische und statistische Daten zu den psychischen Krankheiten kennen und heranziehen, aber es ist genauso wichtig, dass Politiker und Versicherer sich darüber im klaren sind, dass jede Behandlung einen Einzelfall darstellt (was sehr einfach nachzuvollziehen ist - ein jeder mag sich an seine Situation als Patient erinnern. Wäre es uns recht, wenn der Behandelnde uns als statistische Entität behandeln würde?). Hierin liegt wohl die grösste Schwierigkeit eines derartigen Projekts zur Förderung und Verbesserung der psychiatrisch-psychotherapeutischen Behandlung: die Begrenzungen dieser beiden Logiken sollen miteinander verknüpft und nicht einander gegenübergestellt werden. Wichtig ist, dass sich Politik, Versicherer und Behandelnde dessen bewusst sind.

Wie im Expertenbericht vorgeschlagen, geht es darum, ein System zu erarbeiten, zu realisieren und zu fördern, in dem Verschiedenartigkeit und Besonderheit miteinander in Einklang gebracht werden, und das sich nicht wegen allzu idealer Kriterien erschöpft. Ein solches System muss zukunftsorientiert sein und den ständigen Generationenwechsel berücksichtigen, der es immer wieder mit den gleichen Determinanten von psychischer Stabilität/Instabilität konfrontieren wird. Die Vielfalt von Aufnahmestrukturen, die Vielzahl der Versorgungs- und Behandlungsmöglichkeiten usw. müssen von einer Politik für Gesundheit und psychiatrisch-psychotherapeutische Versorgung gefördert werden. Nur so ist es möglich, auf die Einzigartigkeit des Einzelfalles und die globale Verwaltung des Systems einzugehen. Der Einzelfall schliesst einen erweiterten Blick auf die Gesamtsituation nicht aus, insbesondere was die Prozesse mit Blick auf Information, Finanzierung und die Vernetzung der Strukturen angeht.

Im Bericht wird zu Recht darauf hingewiesen, dass es im aktuellen System qualitativ sehr hochwertige Behandlungseinrichtungen und -angebote gibt. Gleichzeitig verweist er aber auch auf die Lücken - insbesondere im Bereich der Früherkennung und der frühzeitigen Behandlung schwerer Entwicklungs- und Beziehungsstörungen in allen Bevölkerungsgruppen. Zudem wird auf das Fehlen von Koordinierungsmassnahmen und Verbindungen zwischen diesen Strukturen und dem Behandlungsangebot verwiesen. Eine der sichtbaren Folgen ist laut Bericht das verzögerte Erkennen von Störungen und der verzögerte Zugang zur Behandlung. Dieser Verzug hat seine Wurzeln auch im Ideal von Leistungsstärke und Effizienz, das in unserer Gesellschaft ständig propagiert wird. In vielen Fällen kann es somit psychisch sinnvoller sein, dem Druck des Ideals des Systems zu entgehen, indem man ein Symptom wählt bzw. ein Defizit entwickelt, das möglicherweise zu einer Rente, einer Entschädigung oder einem anderen Mehr- 
wert führt, das aber vor allem dem Subjekt ermöglicht, etwas von seiner Identität und seinen (wenn auch krankhaften) Identifikationen $\mathrm{zu}$ bewahren. Diese die Ausrichtung unserer Gesellschaft betreffenden Parameter müssen ebenfalls berücksichtigt werden, wenn es um die Wahl von Massnahmen geht, die nach der Lektüre des Berichts erfolgen sollen, da wir ansonsten Gefahr laufen, mit gutem Gewissen hinzunehmen, dass aktuelle und zukünftige Bestandesaufnahmen eine weiterhin prekäre Situation darstellen.

\section{Gesundheitspolitik: Verbesserung der Qualität der Behandlungsangebote in der Allgemeinmedizin sowie in der Psychiatrie und Psychotherapie ${ }^{8}$}

Die verschiedenen, im vorliegenden Bericht angeführten Vorschläge zur Versorgung basieren auf dem Bemühen um eine Definition des Begriffs «psychische Gesundheit» sowie auf der Beschreibung seiner Determinanten: "Das biopsycho-soziale Erklärungsmodell und der salutogenetische Ansatz bilden die theoretischen Grundlagen der vorliegenden Strategie.» (S. 25).

Der auf der Grundlage des Konzepts eines bio-psycho-sozialen Ansatzes basierende Artikel von Engel ist sicherlich interessant. Allerdings ist hier anzumerken, dass man aufgrund dieses Konzepts allzu leicht denken mag, wir verfügten über eine globale Theorie zur psychischen Störung, mit anderen Worten über einen wissenschaftlichen Rahmen, der eine wissenschaftlich begründete Logik des Defizits ermöglicht und legitimiert und für den es eine angemessene und schlüssige Methode gibt. Wir sind jedoch weit davon entfernt, die verschiedenen biologischen, psychologischen und sozialen Determinanten der psychischen Krankheit miteinander verknüpfen und integrieren zu können. Schon allein der genetische Ansatz zeigt, wie wichtig das Individuum und der Einzelfall sind und dass es unmöglich ist, von einer allgemeinen Theorie auf den einzelnen Patienten als Person zu schliessen. Die Tatsache an sich, dass sich die aktuellen Diagnostikregelwerke DSMIVR oder ICD-10 im wesentlichen als atheoretisch verstanden wissen wollen, zeigt, dass es unmöglich ist, sich auf eine einheitliche und sich nicht widersprechende Definition der psychischen Störung zu beziehen.

Das bio-psycho-soziale Modell entspricht grundlegend einem Ansatz, der in der ersten Hälfte des 20. Jahrhunderts, ausgehend von den Arbeiten des in die USA emigrierten schweizeri- schen Psychiaters Adolphe Meyer, vorherrschte. Das aktuelle Modell kann nur ein komplexes Modell sein, im Rahmen dessen der Platz des Individuums und der psychischen Störung, unter der der Patient leidet, ständig neu in Erinnerung gerufen werden muss, um nicht aufgerieben $\mathrm{zu}$ werden zwischen genetischem Determinismus und sozialem und ökonomischen Kontext.

Die Gefahr, dass das Individuum und sein psychisches Leiden untergehen, kommt im Bericht deutlich zum Ausdruck. Im Bericht werden die ausschliesslich medizinischen Aspekte des psychischen Determinismus lediglich gestreift; auf die sozioökonomischen, soziokulturellen und institutionellen Faktoren wird jedoch detailliert eingegangen. Im Bemühen, eine Nationale Gesundheitspolitik zu gestalten, ist dies sicherlich legitim; wenn es aber um den Platz des Patienten in seiner Einzigartigkeit geht und darum, den Sinn des Symptoms für ihn selbst $\mathrm{zu}$ hinterfragen, ist ein solcher Ansatz fraglich. Eine wirklich salutogenetische Perspektive muss unserer Ansicht nach die Beziehung des Subjekts $\mathrm{zu}$ seinem Leiden und seiner psychiatrischen Störung in deren ganz einzigartigen Dimension berücksichtigen.

Eine stärker wissenschaftlich ausgerichtete Argumentation muss die Schlüsselstellung der Psychopathologie in der psychiatrischen und psychotherapeutischen Forschung hervorheben. Die Art, wie sich die Symptome beim Patienten - bei jedem Patienten - äussern, ist ausschlaggebend für jegliche Reflexion, mit der man Biologie, Psyche und Soziales miteinander verknüpfen will. Wird die Betonung zu sehr auf den genetischen, epidemiologischen, kulturellen, sozialen oder gemeinschaftlichen Ansatz gelegt, besteht die Gefahr, dass der Platz der einzelnen Person, jedes Kranken als Ort, an dem sich sämtliche Determinanten der psychischen Störung vereinen, vergessen wird. Möglicherweise eine andere Art zu sagen, dass es keine psychische Störung ohne Kranken und ohne Behandelnden gibt ...

Die im Bericht entwickelte diesbezügliche Strategie führt insbesondere $\mathrm{zu}$ den unter Punkt 6.8 aufgeführten Empfehlungen. Die Gefahr einer übertriebenen Koordination, die zu einer rigiden und unflexiblen Planung führen würde, wird klar ausgeglichen durch die Betonung von Flexibilität und Pluralität hinsichtlich der Versorgung. Es sollte jedoch nicht ausser acht gelassen werden, dass sich diese Aspekte a priori widersprechen. Die Logik der psychischen Störung als Defizit und die wissenschaftsgläubige Illusion mit Blick auf das bio-psycho- 
soziale Modell müssen durch Berücksichtigung der Psychopathologie sowohl auf klinischer Ebene wie auch in der Forschung ausgeglichen werden. Die Psychopathologie berücksichtigen heisst auch, der Originalität und der Kreativität der Patienten und der Behandelnden einen möglichst grossen Spielraum zu lassen in der Weise, mit der sie an die Probleme, die sich aus den psychischen Störungen ergeben, herangehen.

\section{Gesundheitspolitik bedeutet auch, die Wahl der Gesellschaft zu hinterfragen, das Bestehende besser zu steuern, den Zugang und das Angebot im Bereich der Versorgung zu optimieren und die Behandlung nicht durch unangebrachtes Intervenieren von seiten der Politiker und Versicherer zu erschweren}

Im Bericht wird von einem allgemeinen «Steuerungsbedarf» (S. 65) gesprochen. Hierbei wird zu Recht auf die Notwendigkeit eines Gremiums, eines multidisziplinären Organs, hingewiesen, mittels dessen - ausgehend von der Arbeit vor Ort - die Bedürfnisse, die Mittel und die Prioritäten im Bereich Versorgung und Proportionalität bezüglich psychischer Gesundheit festgelegt werden könnten.

Im Teil B des Berichts wird bezüglich des Aktionsplans vorgeschlagen, mit Blick auf die stets gleiche Problematik eine sich ständig erneuernde Strukturenvielfalt zu schaffen; damit wird jedoch die notwendige Flexibilität des Systems eingeschränkt. Die in diesem Teil eingebrachten Empfehlungen sind zwar, was das Ziel angeht, alle zutreffend, in ihrer Anwendung jedoch zu komplex. In dieser Form kommen zu viele Fragen auf und die Arbeit der Akteure vor Ort wird erschwert. Es droht eine bürokratische Maschinerie, die die konkrete Arbeit vor Ort unnötig übergehen und verteuern würde.

Damit erscheint uns die Idee, auf nationaler Ebene ein Steuerungsorgan einzurichten, der Weg zu sein, der am dringendsten einzuschlagen wäre. Um einwandfrei funktionieren zu können, müsste dieses Steuerungsorgan eine ständige, vielseitige, legitimierte und glaubwürdige Einrichtung sein. Seine Aufgabe wäre es, die Benützer, Politiker, Leistungserbringer und Versicherer für die Synergien, aber auch für die spezifischen Entwicklungen und die Prioritäten, die im Bereich der psychischen Gesundheit und Versorgung $\mathrm{zu}$ verwirklichen sind, $\mathrm{zu}$ sensibilisieren.
Die Legitimation dürfte sich dabei nicht allein auf der Präsenz politischer Organe gründen, wie dies möglicherweise ein wenig zu stark in Teil B betont wird, sondern auf das Vorhandensein einer repräsentativen Vertretung der im betroffenen Bereich tätigen Akteure. ${ }^{9}$ Ein solches Steuerungsorgan sollte sich nach individueller und kollektiver Verantwortung, Kompetenz, Qualität, Transparenz und Vielfalt (Pluralität) ausrichten.

\section{Schlussfolgerung. Für und nicht gegen unsere unauflösbare Ambivalenz bezüg- lich psychischer Phänomene arbeiten}

Auf Bundes- und Kantonsebene ist man sich der Bedeutung der psychischen Dimension für die Lebensqualität der schweizerischen Bevölkerung bewusst geworden, doch sollte man sich nicht zu schnell über diesbezügliche vermeintliche Auswirkungen freuen. Es gibt einige aktuelle Indikatoren, die zeigen, dass es bei diesem Bewusstwerden mit Blick auf die notwendige Verbesserung der psychischen Gesundheit in unserer Bevölkerung durchaus Abstufungen gibt. Die Ambivalenz gegenüber dem Psychischen zeigt sich in einer Tendenz zum Ausweichen, zur Feindseligkeit sowie in destruktiven Massnahmen an verschiedenen Schauplätzen: das neue Gesetz über die lebenslängliche Haft von sogenannt gefährlichen Straftätern, die Diskriminierung von Personen, die aus psychischen Gründen einen Arzt aufsuchten, durch die Versicherungen, das Infragestellen der Psychotherapie durch einige politische Parteien, die Schliessung von psychischen Behandlungseinrichtungen trotz fehlendem Nachweis von Unwirtschaftlichkeit bzw. Nichteffizienz, die fehlende Ermutigung zur Ausbildung in psychiatrischer Krankenpflege usw.

Der Ursprung dieser Ambivalenz gründet wohl auch in der Tatsache, dass die Spannung zwischen dem Notwendigen und der rechnungspflichtigen Logik der Krankenversicherer die Politiker heute dazu drängt, aus den Methoden und Werkzeugen der heutigen Psychiatrie/Psychotherapie ausschliesslich die zu ihrem Wirtschaftlichkeitsprojekt passenden Instrumente herauszusuchen und $\mathrm{zu}$ selektionieren. Im Bereich des Psychischen spielt sich das alles unter dem Deckmantel einer falschen wissenschaftlich begründeten Universalität und - vor allem - einer Allmachtsphantasie ab. Die Psychiatrie/Psychotherapie muss diesbezüglich wachsam sein, wenn sie nicht Gefahr laufen will, zur Verarmung des sozialen Gefüges beizutragen. 
Abschliessend können wir somit die im Bericht vorgeschlagenen spezifischen Entwicklungen im Bereich der Psychiatrie/Psychotherapie (Früherkennung und frühe Behandlung) sowie die Förderung der psychischen Gesundheit nur unterstützen. In unserer Analyse sprechen wir uns ganz besonders für die Einrichtung eines multidisziplinären, ständigen Koordinationsorgans aus, mit dessen Hilfe vielfältige Behandlungsangebote und -methoden beibehalten und gefördert werden sollen.

Dies ist nach unserer Einschätzung das wirtschaftlich sinnvollste Mittel, um Gesundheitsförderung einerseits, ein angemessenes Verhältnis zwischen Versorgung des einzelnen und Bedürfnissen der Gemeinschaft andererseits zu erreichen.

\section{Anmerkungen}

1 Die FMPP ist der Dachverband der Psychiater/-innen und Psychotherapeuten/-innen. Ihr gehören Kinderund Jugendpsychiater/innen und -psychotherapeuten/ -innen sowie Fachärztinnen/-ärzte für Erwachsenenpsychiatrie und -psychotherapie der SGPP an. Amtierender Präsident ist Dr. H. Kurt.

2 Ständige Kommission für Strategie und Planung Psychiatrie und Psychotherapie.

3 Foederatio Medicorum Psychiatricorum et Psychotherapeuticorum.

4 Schweizerische Gesellschaft für Psychiatrie und Psychotherapie.

5 Der Konjunktiv ergibt sich hier aus der Ambivalenz dieses Interesses für den psychischen Bereich. Siehe «Schlussfolgerung».

6 In der Funktionsweise des psychischen Apparates gehört zum Vokabular der Psychoanalyse auch der Begriff der Ökonomie.

7 Das Subjekt in die Verantwortung einbeziehen, bedeutet, nicht ihm die Schuld zu geben, wenn es nicht oder nicht mehr wirtschaftlich aktiv ist. Durch ein In-die-Verantwortung-Nehmen soll das Subjekt darin unterstützt werden, im Rahmen der ihm zur Verfügung stehenden Mittel die Auswirkungen dessen zu tragen, was es wünscht.

8 Dieses Kapitel wurde von Professor Jean-Nicolas Despland, DUPA, Lausanne, verfasst.

9 Das Steuerungsorgan könnte sich beispielsweise aus Erbringern psychischer Behandlungsleistungen (FMH, FMPP, klinische Psychologie), Versorgungsstrukturen (Sozialdienste usw.), Politik (Bund: EDI, BAG, BSV, Obsan, BFS, KLV, IV und Kantone: SODK), der Wirtschaft (santésuisse) und Nutzern zusammensetzen. 